\title{
Formalin-induced pain prolongs sub- to supra-second time estimation in rats
}

\author{
Xinhe Liu ${ }^{1,2}$, Ning Wang ${ }^{\text {Corresp., } 1,2}$, Jinyan Wang ${ }^{1,2}$, Fei Luo ${ }^{1,2}$ \\ ${ }^{1}$ CAS Key Laboratory of Mental Health, Institute of Psychology, Chinese Academy of Sciences, Beijing, China \\ 2 Department of Psychology, University of Chinese Academy of Sciences, Beijing, China \\ Corresponding Author: Ning Wang \\ Email address: wangn@psych.ac.cn
}

Background: Temporal estimation can be influenced by pain, which is a complex psychological and physiological phenomenon. However, the time range in which perception is most sensitive to pain remains unclear. Methods: In the present study, we explored the effects of acute inflammatory pain on time perception in the sub- to suprasecond (0.6-2.4-s) and supra-second (2-8-s) ranges in rats. Plantar formalin injection was used to induce acute inflammatory pain, and a temporal bisection task was used to measure time perception. Task test sessions were held for five consecutive days (one per day): the day before injection (baseline), immediately after injection, and the three postinjection days. The point of subjective equality (PSE, which reflects the subjective duration) and Weber fraction (which reflects temporal sensitivity) were calculated and analysed.

Results: In the 0.6-2.4-s range, the PSE was significantly lower, indicating prolonged subjective duration, in the formalin group relative to the saline group $(p=0.049)$ immediately after injection. Formalin-induced pain also tended to lengthened time perception in the $0.6-2.4$-s range on post-injection days $2(p=0.06)$ and $3(p=0.054)$. In the 2-8-s range, formalin injection did not affect the PSE or Weber fraction. Conclusions: The enhanced effect of pain on temporal perception in the sub- to supra-second range is observed in this study and this effect is attenuated with the prolongation of estimated time, even in rats. 
1

2

3

4

5 1. CAS Key Laboratory of Mental Health, Institute of Psychology, Chinese Academy of

6 Sciences, Beijing, 100101, P.R. China.

7 2. Department of Psychology, University of Chinese Academy of Sciences, Beijing, 100049,

8 P.R. China.

9

10

11 12

13

14

\section{Formalin-induced pain prolongs sub- to supra-second time estimation in rats}

Xinhe LIU ${ }^{1,2}$, Ning WANG ${ }^{1,2 *}$, Jinyan WANG ${ }^{1,2}$, Fei LUO ${ }^{1,2}$

*Corresponding Author information:

Ning WANG, Ph.D. CAS Key Laboratory of Mental Health, Institute of Psychology, Chinese

Academy of Sciences, 16 Lincui Road, Chaoyang District, Beijing, 100101, P.R. China. E-mail:

wangn@psych.ac.cn 
15

16 Abstract

17 Background: Temporal estimation can be influenced by pain, which is a complex psychological

18 and physiological phenomenon. However, the time range in which perception is most sensitive to 19 pain remains unclear.

20 Methods: In the present study, we explored the effects of acute inflammatory pain on time 21 perception in the sub- to supra-second (0.6-2.4-s) and supra-second (2-8-s) ranges in rats.

22 Plantar formalin injection was used to induce acute inflammatory pain, and a temporal bisection 23 task was used to measure time perception. Task test sessions were held for five consecutive days 24 (one per day): the day before injection (baseline), immediately after injection, and the three post25 injection days. The point of subjective equality (PSE, which reflects the subjective duration) and 26 Weber fraction (which reflects temporal sensitivity) were calculated and analysed.

27 Results: In the 0.6-2.4-s range, the PSE was significantly lower, indicating prolonged subjective 28 duration, in the formalin group relative to the saline group $(p=0.049)$ immediately after 29 injection. Formalin-induced pain also tended to lengthened time perception in the 0.6-2.4-s 30 range on post-injection days $2(p=0.06)$ and $3(p=0.054)$. In the $2-8$-s range, formalin injection 31 did not affect the PSE or Weber fraction.

32 Conclusions: The enhanced effect of pain on temporal perception in the sub- to supra-second 33 range is observed in this study and this effect is attenuated with the prolongation of estimated 34 time, even in rats.

35 Keywords: Time perception; Formalin-induced pain; Temporal bisection task; Temporal range 
36

37

38

39

40

41

42

43

44

45

46

47

48

49

50

51

52

53

54

55

56

57

58

59

60

61

62

63

64

65

66

67

68

69

70

71

72

73

74

75

\section{Introduction}

Pain plays an important role in protecting individuals from potential or further injury (Baliki \& Apkarian 2015). Stress and anxiety often accompany acute pain, which has short-term impacts on attention, motivation and decision making (Wiech 2016; Wiech \& Tracey 2013). A growing body of evidence suggests that acute pain influences time perception. Human laboratory studies have shown that healthy subjects overestimate the durations of painful stimuli (Khoshnejad et al. 2017b; Piovesan et al. 2019) and neutral stimuli paired with painful stimuli (Hare 1963; Ogden et al. 2015). In the context of acute pain, healthy subjects experienced a prolonged sense of neutral signalling (Ogden et al. 2015; Rey et al. 2017).

Considerable number of evidences suggest that the perception of short but not long duration is modulated by negative emotional stimulus; the greatest effect has been observed for short-term (2-s) time perception, with a gradual decrease with the prolongation of duration (to 4-6 s) (Angrilli et al. 1997; Noulhiane et al. 2007). The reason for this phenomenon may be that emotional stimuli influence time perception from various psychological processes. The classic pacemaker-accumulator model entails the assumption that working memory, reference memory and arousal are involved in time perception (Gibbon et al. 1984; Treisman 1963). Noulhiane et al (2007) and Treisman (1963) suggested that emotional stimulus-induced high arousal increases the internal clock rate. Some studies have shown that this arousal only affects the processing of short duration (no more than 3-4 s) (Angrilli et al. 1997; Noulhiane et al. 2007).

As a complex physiological and psychological phenomenon with sensory, emotional and cognitive components (Melzack \& Wall 1965), pain has been found to increase physiological arousal levels (Barr 1998; Santuzzi et al. 2013). Therefore, pain might modulate time perception (or clock) only in short duration. However, a notable problem is most studies of the effect of acute pain on time perception have focused on short (<2.4-s) temporal durations (Liu et al. 2019; Ogden et al. 2015; Rey et al. 2017). Thus, our understanding of time processing in the presence of pain remains limited. Therefore, it is important to clarify the effect of pain on long as well as short temporal range.

In human studies, long temporal ranges are affected easily by strategies such as counting (Thönes \& Hecht 2017). In order to know the effects of pain on longer temporal range, using animal is helpful because animal does not use such strategy. They are thus useful for performing time perception tasks over long temporal ranges (Brown et al. 2011; Kamada \& Hata 2018; Kamada \& Hata 2019; Orduña et al. 2011). Temporal bisection task is a classic paradigm of time perception, which is used widely in animals (Brown et al. 2011; Church \& Deluty 1977; Deane et al. 2017; Kamada \& Hata 2018; Kamada \& Hata 2019; Liu et al. 2019; Meck 1983; Soares et al. 2016) and humans (Fayolle et al. 2015; Huang et al. 2018; Rey et al. 2017; Tipples 2011). In this study, we used the temporal bisection task to investigate how acute inflammatory pain modulates time perception over shorter (sub- to supra-second range, 0.6-2.4 s) and longer (supra-second or second to minute range, 2-8 s; (Kamada \& Hata 2018; Kamada \& Hata 2019)) temporal ranges in rats. The acute inflammatory pain was induced by subcutaneous injection of formalin into the hind paw, and the rats were trained to perceive temporal durations and classify 
76 them and long and short in the temporal bisection task. The experimental data obtained were

77 fitted with a theoretical model, and the point of subjective equality (PSE, a measure of subjective

78 duration) and Weber fraction (a measure of temporal sensitivity) within the temporal ranges

79 examined were calculated. We also explored post-injection effects on time perception, as

80 formalin-induced pain has been shown to influence animals' emotion-related behaviours even

81 days after injection (Jiang et al. 2014; Johansen et al. 2001).

\section{Materials \& Methods}

83

84

85

86

87

88

89

90

91

92

93

94

95

96

97

98

99

100

101

102

103

104

105

106

107

108

109

110

111

112

\section{Animals}

Thirty-two male Sprague-Dawley rats (weighing 230-250 g on arrival, purchased form Charles River, Beijing, China) were used in this study. The rats were allowed to adapt to the laboratory environment for at least 1 week before the experiment was conducted, and they were handled (by stroking them or letting them move freely on the experimenter's arms for $5 \mathrm{~min}$ ) once per day to familiarise them with manipulation by the experimenter. They were housed individually in separate cages with food and water available ad libitum; but since the rats were able to get water from the task, we removed the water bottle from the feeding cage the day before the formal experiment. When a rat did not perform the task on any day, it was allowed to drink freely from a standard 250-ml bottle for $5 \mathrm{~min}$. In addition, we recorded the rats' body weights every 3-4 days and gave them an additional $5 \mathrm{~min}$ access to water when a loss of $>10 \mathrm{~g}$ from the last recording was noted. During the experimental period, the rats' body weights were maintained between 270 and $360 \mathrm{~g}$.

The room in which the animals were housed was temperature and humidity controlled (22 $\pm 2^{\circ} \mathrm{C}, 50 \% \pm 10 \%$ humidity), with a 12/12-h light/dark cycle (lights on at 8:00 pm). All experiments were performed in accordance with the National Institutes of Health guidelines for the care and use of laboratory animals (ISBN: 0-309-05377-3, First Printing, 1996) and approved by the Institutional Review Board of the Institute of Psychology, Chinese Academy of Sciences. The approve number is H16036. All rats were alive upon completion of this research and were used in preliminary experiments for another study.

\section{Experimental apparatus}

Temporal discrimination training and temporal bisection testing of each rat were conducted in the same operant box $(21 \mathrm{~cm} \mathrm{~W} \times 32.5 \mathrm{~cm} \mathrm{~L} \times 42.5 \mathrm{~cm} \mathrm{H})$ located in a soundproof chamber. The box was made of acrylic, had a strip floor and was positioned above a waste catch pan. Two retractable levers (ENV-112CMP; MED Associates Inc., St. Albans, VT, USA) were mounted symmetrically on one wall of the box, $9 \mathrm{~cm}$ above the floor. A liquid dispenser (ENV-201A) and water receptacle ( $2.5 \mathrm{~cm}$ above the floor) were spaced equally between the levers to provide water as the reinforcer. As the to-be-timed auditory cue, a pure-tone stimulus $(2900 \mathrm{~Hz}, 65 \mathrm{~dB})$ was presented by a tone generator (ENV-223AM) mounted on the wall above the liquid dispenser. An illuminated infrared detective nose-poke hole (ENV-114BM) was mounted in the

Peer) reviewing PDF | (2020:08:52137:2:0:NEW 20 Jan 2021) 
113 middle of the wall opposite the levers, $2.5 \mathrm{~cm}$ above the floor. An indicator lamp (ENV-221M) 114 was located directly above the nose-poke hole. An exhaust fan was mounted on the soundproof 115 chamber. The operant box was connected to a computer that recorded the rats' experimental 116 events. The experimenter furnished all output and input devices mounted in the box. The box 117 was cleaned with water and medical-grade alcohol after each session.

\section{Experimental procedure}

The temporal bisection task was conducted with 16 rats each for the sub- to supra-second and supra-second ranges. After training, each rat was first submitted to three test sessions (one per day) to stabilise its performance. Then, each rat performed five test sessions on five consecutive days (one per day): the day before formalin injection (baseline), immediately after formalin injection, and 1-3 days after formalin injection. Before the day of injection, the rats were assigned to formalin and saline control groups ( $n=8$ each) according to their baseline performance. All experiments were performed during the rats' light cycle.

\section{Temporal discrimination training}

Temporal discrimination training was performed using a procedure modified according to previous research (Brasted et al. 1997; Callu et al. 2009; Deane et al. 2017). It consisted of leverpress training (1-2 days), forced-choice training ( 3 days), and free-choice response training (12 days). In each trial of a lever-press training session (1-h duration, no limit on trial number), an anchor-duration tone was presented, and only the corresponding lever was extended. The anchorduration tone was the short or long tone used for conditioning ( 0.6 or $2.4 \mathrm{~s}$ for the sub- to suprasecond range and 2 or $8 \mathrm{~s}$ for the supra-second range). For the forced-choice training trials $(n=$ 100 , equal numbers with long and short anchor durations), each tone was triggered by a rat's nose-poke behaviour. Only the corresponding lever was extended following the termination of the tone. For the free-choice response training trials $(n=100)$, the tones were triggered by the rats' nose-poke behaviours, and their termination was followed by the simultaneous extension of both levers. On the first 6 days of free-choice training, the levers were retracted only after a rat provided the correct response; on the next 6 days, the levers were retracted immediately after any response. Correct responses were followed by the provision of drips of water as a reinforcer. Non-response for $5 \mathrm{~s}$ resulted in lever retraction and no reward. The criterion for the successful completion of temporal discrimination training was the achievement of correct response rates $\geqslant$ $85 \%$ on 3 consecutive days. For all training and testing sessions, the rats were allowed to move freely in the operant box.

\section{Temporal bisection testing}

During the testing stage of the temporal bisection task, a series of to-be-timed tones of anchor and intermediate durations was presented, and the rats were free to press any of the levers. In the testing sessions, each trial began with the illumination of the indicator light. The to-be-timed tone was initiated by a nose poke. At the end of the tone, the two levers were extended simultaneously. Lever retraction was prompted by any lever press or non-response for $5 \mathrm{~s}$. The 
151

152

153

154

155

156

157

158

159

160

161

162

163

164

165

166

167

168

169

170

171

172

173

174

175

176

177

178

179

180

181

182

183

184

185

186

187

188

average intertrial intervals (measured from lever retraction to the beginning of the next trial) per rat and session were $20 \mathrm{~s}$ (range, 19-21 s) for the 0.6-2.4-s range and $17 \mathrm{~s}$ (range, 13-19 s) for the $2-8$-s range. One testing session consisted of 140 trials $(30 \mathrm{~s} /$ trial $): 14$ trials each with tones of five intermediate durations without reinforcement, and 35 trials each with the two anchorduration tones with reinforcement. Typically, the rats obtained about 65 reinforcements per testing session. For the sub- to supra-second-range (0.6-2.4-s) experiment, the five intermediate durations were set at $0.756,0.952,1.2,1.51$ and 1.9 s. For the supra-second range (2-8-s) experiment, the intermediate durations were set at 2.51, 3.17, 4, 5.04 and $6.35 \mathrm{~s}$. The sequence of these trials was random, but with the constraint of no more than three consecutive intermediateduration trials.

\section{Establishment of pain model}

Acute inflammatory pain was induced by injection of $50 \mu 11 \%$ formalin solution into the plantar surface of each rat's hind paw. An equal-volume saline injection was used as the control. After injection, the rats were returned to the operant box to perform the bisection task, and their nociceptive behaviours (paw licking and paw lifting) during the 70-min test sessions were video recorded. The durations of paw lifting and licking, measured by the experimenter in 5-min epochs, and the cumulative duration of nociceptive behaviours in phases I (0-10 min) and II (20$60 \mathrm{~min}$ ) were calculated for analysis.

\section{Data analysis}

\section{Curve fitting}

The proportion of "long" responses $\left(P_{\mathrm{L}}\right.$, calculated by dividing the number of responses to the "long" lever by the total number of responses in trials with the same tone duration) to each to-betimed tone in each temporal bisection session was used to analyse time perception. Curve fitting and parameter calculation were performed using the Prism software (version 5; GraphPad Software Inc., San Diego, CA, USA). Following previous work (Deane et al. 2017; McClure et al. 2005; Ward \& Odum 2007), the final fitting curve is a cumulative Gaussian distribution function:

$$
F(t)=a+\frac{b}{\sigma \sqrt{2 \pi}} \int_{-\infty}^{t}\left[\exp -\left(\frac{(t-\mu)^{2}}{2 \sigma^{2}}\right)\right] \mathrm{d} t
$$

where $F(\mathrm{t})$ is the $P_{\mathrm{L}}$ when the duration is equal to a given sample $(t), \mu$ is the mean, and $\sigma$ is the standard deviation (representing the slope of the function). Parameters $a$ represents the low asymptote and $b$ represents the range of function. The mean $(\mu)$ is also the PSE, defined as a $50 \%$ chance that the animal will provide a "long" response $\left(P_{\mathrm{L}}=50 \%\right)$. The PSE reflects the subjectively perceived length of time, with increases and decreases therein interpreted as underand overestimation, respectively, of the duration (Kamada \& Hata 2018; Kamada \& Hata 2019; Rey et al. 2017). The Weber fraction, an index of temporal sensitivity, was calculated by dividing the standard deviation by the PSE (Crystal et al. 2003). A decrease in the Weber 
189 fraction indicates an increase in temporal sensitivity (Deane et al. 2017; McClure et al. 2005;

190

191

192

193

194

195

196

197

198

199

200

201

202

203

204

205

206

207

208

209

210

211

212

213

214

215

216

217

218

219

220

221

222

223

224

225

226

Ward \& Odum 2007).

The $R^{2}$ statistic was used to quantify the goodness of fit. For each testing session, an individual rat's $P_{\mathrm{L}}$ was fitted with this model to obtain the PSE and Weber fraction (Fig. 1F, G, etc); the average $P_{\mathrm{L}}$ of each group of rats was fitted with this model to obtain the best-fitting curve (Fig. 1D, E, etc). When $R^{2}$ was $<0.7$ in curve fitting for any individual rat, the PSE and Weber fraction were considered to be inaccurate and were discarded from the analysis.

\section{Statistical analysis}

To assess the post-treatment effect on the PSE, we calculated the mean PSE and Weber fraction, and calculated the change in the PSE relative to baseline using the following formula: change in PSE $=[$ (current-session PSE - baseline PSE $) /$ baseline PSE $)] \times 100 \%$. Changes in the Weber fraction in the post-injection sessions were calculated using the same formula. The Prism (version 5; GraphPad Software Inc.) and SPSS (version 25; IBM Corporation, Armonk, NY, USA) software packages were used for graph generation and statistical analyses, respectively. Two-way repeated measure analysis of variance (two-way RM ANOVA) followed by a simple main-effect analysis was conducted to analyse the following variables: nociceptive behaviours (group * time); $P_{\mathrm{L}} \mathrm{S}$ and response latency for each duration in the temporal bisection task [(group * duration) or (day* duration)]; PSE, Weber fraction and omitted trials at baseline and on the injection day (group * day). Independent-sample $t$ test was used to compare the difference of the cumulative duration of paw licking and lifting, the daily correct response rates, and the omitted trials and the change in PSE/Weber fraction on each post-injection day between the two groups. If these data do not conform to the assumptions of the statistical method, we will use the correction method. The data are expressed as means \pm standard errors of the mean, and the significance level was set at $\alpha<0.05$. Data from trials with no lever-press response were omitted from the analysis.

\section{Results}

\section{Effect of formalin-induced pain on temporal estimation in the sub- to supra- second range}

As shown in Fig. 1A, formalin injection induced a typical biphasic pattern of nociceptive behaviours. Compared with the saline group, the formalin group exhibited significantly more such behaviours during the temporal bisection task [interaction of treatment and time: $F(13$, $182)=8.624, p<0.001$; treatment effect: $F(1,14)=28.500, p<0.001$; time effect: $F(13,182)$ $=10.844, p<0.001$; Fig. 1A]. Cumulative duration of paw licking and lifting after formalin injection were increased significantly in phases I $[t(14)=-5.711, p<0.001$; Fig. 1B] and II $[t$ $(14)=-4.433, p<0.001$; Fig. 1C] compared with those after saline injection.

We compared the correct response rates to anchor-duration tones between two groups at baseline (0.6 s: saline $96.75 \% \pm 1.38 \%$, formalin $96.07 \% \pm 1.20 \% ; 2.4 \mathrm{~s}$ : saline $94.29 \% \pm 2.96 \%$, formalin $95.34 \% \pm 3.10 \%)$ and on the injection day $(0.6 \mathrm{~s}$ : saline $90.71 \% \pm 3.23 \%$, formalin

Peer] reviewing PDF | (2020:08:52137:2:0:NEW 20 Jan 2021) 
227

228

229

230

231

232

233

234

235

236

237

238

239

240

241

242

243

244

245

246

247

248

249

250

251

252

253

254

255

256

257

258

259

260

261

262

263

264

265

$90.57 \% \pm 3.19 \%$; 2.4 s: saline $96.01 \% \pm 2.21 \%$, formalin $97.37 \% \pm 1.05 \%$ ), respectively. Independent-sample $t$ tests revealed no difference in the correct response rate at baseline or on the injection day, indicating that formalin treatment did not affect the animals' temporal discrimination ability.

The effect of formalin injection on $P_{\mathrm{L}} \mathrm{S}$ of temporal bisection task was presented in Fig. 1D, E. Two-way RM ANOVA revealed no significant interaction between duration and treatment affecting the $P_{\mathrm{L}}$ at baseline $[F(6,84)=0.489, p=0.815$, Fig. 1D] and on the injection day $[F$ $(6,84)=2.486, p=0.23$, Fig. 1E]. Nevertheless, the significant duration effect showed that the rats could discriminate the durations of the to-be-timed tones $(p<0.001)$. For each group, the differences of $P_{\mathrm{L}} \mathrm{s}$ between baseline day and injection day were also compared by Two-way RM ANOVA. A significant interaction of the day and duration was found in the formalin group $[F$ $(6,42)=2.486, p=0.038]$ and a simple main-effect analysis showed that $P_{\mathrm{L}} \mathrm{S}$ on the injection day were higher than these at baseline at $0.952 \mathrm{~s}(p=0.030)$ and $1.2 \mathrm{~s}(p=0.017)$. No such effect was observed in the saline group $[F(6,42)=0.790, p=0.583]$. $R^{2}$ values for curve fitting ranged from 0.86 to 0.99 (average, 0.94 ) in the two groups at baseline and on the injection day, with no difference between groups.

As presented in Fig. 1F, two-way RM ANOVA revealed significant interaction of the treatment and day affecting PSE values $[F(1,14)=6.405, p=0.024]$. A simple main-effect analysis showed that the PSE was lower in the formalin group than in the saline group on the injection day $[F(1,14)=4.67, p=0.049]$, but not at baseline $[F(1,14)=0.02, p=0.899]$. The Weber fraction, however, did not change significantly [interaction of treatment and day: $F$ (1, 14) $=0.017, p=0.897$, Fig. $1 \mathrm{G}]$. Moreover, we found a negative correlation between the total duration of nociceptive behaviours and the PSE on the injection day in all rats $(r=-0.505, p=$ 0.046, Pearson product-moment correlation; Fig. 1H).

We also compared the omitted trials at baseline (saline, $4.125 \pm 3.199$; formalin, $0.875 \pm$ 0.372 ) and on the injection day (saline, $4.250 \pm 2.296$; formalin, $3.750 \pm 0.862$ ). Two-way RM ANOVA revealed that the treatment had no significant effect in the omitted trials [treatment effect: $F(1,14)=0.400, p=0.537$; interaction of day and treatment: $F(1,14)=3.264, p=$ 0.092]. The response latency (defined as the time between lever extension and pressing) did not differ between groups at baseline [treatment effect: $F(1,14)=2.140, p=0.166$; interaction of duration and treatment: $F(6,84)=1.814, p=0.106$, Supplemental Fig.1A] or on the injection day [treatment effect: $F(1,14)=0.113, p=0.741$; interaction of duration and treatment: $F(6$, $84)=0.693, p=0.656$; Supplemental Fig. 1B].

\section{Effect of formalin-induced pain on temporal estimation in the supra-second range}

In this range, formalin injection also induced a typical biphasic pattern of nociceptive behaviours during the temporal bisection sessions. As shown in Fig. 2A, formalin injection significantly increased nociceptive behaviours compared with saline injection [treatment effect: $F(1,13)=26.183, p<0.001$; time effect: $F(13,169)=11.190, p<0.001$; interaction of 
266 treatment and time: $F(13,169)=7.363, p<0.001$ ]. The cumulative duration of paw licking and 267 paw lifting was significantly greater in the formalin group than in the saline group in phases I $[t$ 268 (13) $=-6.577, p<0.001$; Fig. 2B $]$ and II $[t(13)=-3.155, p=0.019$; Fig. 2C $]$.

269

270

271

272

273

274

275

276

277

278

279

280

281

282

283

284

285

286

287

288

289

290

291

292

293

294

295

296

297

298

299

300

301

302

Correct response rates to anchor durations was also analysed. Independent-sample $t$ tests revealed no significant difference at baseline ( $2 \mathrm{~s}$ : saline $99.28 \% \pm 0.47 \%$, formalin $100.00 \% \pm$ $0.00 \%$; 8 s: saline $97.99 \% \pm 1.05 \%$, formalin $94.67 \% \pm 2.29 \%$ ) or on the injection day ( $2 \mathrm{~s}$ : saline $99.62 \% \pm 0.38 \%$, formalin $95.81 \% \pm 3.74 \%$; 8 s: saline $94.52 \% \pm 1.13 \%$, formalin $97.53 \%$ $\pm 1.16 \%$ ), indicating that formalin treatment did not influence the temporal discrimination ability within this temporal range.

The effect of formalin injection on $P_{\mathrm{L}} \mathrm{S}$ in the supra-second range was presented in Fig. 2D, E. Two-way RM ANOVA revealed no significant interaction of duration and treatment affecting the $P_{\mathrm{L}}$ at baseline $[F(6,78)=0.405, p=0.874]$ or on the injection day $[F(6,78)=0.680, p=$ $0.666]$. However, the treatment had a significant main effect on the injection-day $P_{\mathrm{L}}[F(1,13)=$ $5.186, p=0.040]$; no such effect was observed at baseline $[F(1,13)=0.202, p=0.660]$. The significant duration effect revealed that the rats discriminated the durations of the to-be-timed tones $(p<0.001)$. For each group, two-way RM ANOVA was also used to compare the differences of $P_{\mathrm{L}}$ s between baseline day (Fig. 2D) and injection day (Fig. 2E). No significant interaction of the day and duration was found in the formalin group $[F(6,36)=0.804, p=$ $0.573]$ or saline group $[F(6,42)=0.335, p=0.915]$. $R^{2}$ values for curving fitting ranged from 0.81 to 0.99 (mean, 0.95 ) in the two groups at baseline and on the injection day, with no difference between groups [data from one rat were excluded because the $R^{2}$ values from all tests were low (mean, $0.734 \pm 0.168$ )].

As shown in Fig. 2F, G, no significant interaction of the treatment and day affecting the $\operatorname{PSE}[F(1,13)=0.772, p=0.396]$ or the Weber fraction $[F(1,13)=0.892, p=0.379 ;$ Fig. $2 \mathrm{G}]$ was observed. In addition, we observed no correlation between the PSE and nociceptive behaviours of all rats in the supra-second range ( $r=-0.044, p=0.877$, Pearson product-moment correlation; Fig. 2H).

Two-way ANOVA revealed no significant difference between omitted trials at baseline (saline, $4.571 \pm 1.45$; formalin, $10.875 \pm 3.966$ ) and on the injection day [saline, $7.571 \pm 2.203$; formalin, $10.500 \pm 3.375$; treatment effect: $F(1,13)=1.199, p=0.293$; interaction of treatment and day: $F(1,13)=1.503, p=0.242]$. At baseline, response latency did not differ between groups [treatment: $F(1,13)=0.848, p=0.374$; interaction of duration and treatment: $F(6,78)=$ $1.120, p=0.358$; Supplemental Fig. 2A]. A significant interaction of duration and treatment affected response latency on the injection day $[F(6,78)=4.169, p=0.001]$; no such interaction was observed at baseline $[F(6,78)=0.418, p=0.865$; Supplemental Fig. 2B]. A simple maineffect analysis showed that the formalin treatment prolonged the response latency in the 4-s trial $(p=0.035)$.

Peer) reviewing PDF | (2020:08:52137:2:0:NEW 20 Jan 2021) 


\section{Post-treatment effect of formalin-induced pain on temporal estimation}

304

305

306

307

308

309

310

311

312

313

314

315

316

317

318

319

320

321

322

323

324

325

326

327

328

329

330

331

332

333

334

335

336

337

338

339

340

341

342

The correct response rates of temporal bisection tasks performed in the 0.6-2.4-s range on post-injection days were analysed. Independent-sample $t$ tests revealed no significant difference in the correct response rates to anchor-duration tones on post-injection day 1 ( $0.6 \mathrm{~s}$ : saline $94.94 \% \pm 1.99 \%$, formalin $93.51 \% \pm 3.42 \% ; 2.4 \mathrm{~s}$ : saline $95.89 \% \pm 2.22 \%$, formalin $95.27 \% \pm$ 1.32\%), 2 (0.6 s: saline $95.63 \% \pm 1.46 \%$, formalin: $95.56 \% \pm 2.61 \% ; 2.4$ s: saline $96.05 \% \pm$ $0.93 \%$, formalin $96.04 \% \pm 1.70 \%$ ), or $3(0.6 \mathrm{~s}$ : saline $96.78 \% \pm 1.66 \%$, formalin $97.82 \% \pm$ $1.44 \% ; 2.4 \mathrm{~s}$ : saline $98.21 \% \pm 0.93 \%$, formalin $96.07 \% \pm 1.20 \%$ ). These results indicate that the post-injection effect of formalin treatment did not influence the animals' temporal discrimination ability for the anchor durations.

The results of temporal bisection tasks performed in the $0.6-2.4$-s range on post-injection days are presented in Fig. 3A-C. No significant interaction of treatment and duration affecting the $P_{\mathrm{L}}$ was observed [post-injection day 1: $F(6,84)=1.861, p=0.097$; post-injection day 2: $F$ $(6,84)=1.025, p=0.415$; post-injection day $3: F(6,84)=1.706, p=0.130]$. The main treatment effect also was not significant [post-injection day $1: F(1,14)=1.304, p=0.273$; postinjection day $2: F(1,14)=2.300, p=0.152$; post-injection day $3: F(1,14)=1.359, p=0.263]$. The main effect of duration was significant in all tests [post-injection day $1: F(6,84)=102.703$, $p<0.001$; post-injection day $2: F(6,84)=92.340, p<0.001$; post-injection day $3: F(6,84)=$ $116.652, p<0.001]$, indicating that the rats could discriminate the durations of the to-be-timed auditory stimuli. $R^{2}$ values for curve fitting ranged from 0.87 to 1 (average: 0.96 ), with no significant difference between groups on any of the 3 days.

As shown in Fig. 4A, B, we compare the change in the PSE and Weber fraction in the 0.62.4-s range from baseline on each post-injection day (Original PSE, day 1: saline $1345.375 \pm$ 53.468, formalin $1183.15 \pm 111.639$; day 2 : saline $1413.375 \pm 124.251$, formalin $1140.388 \pm$ 128.744; day 3: saline 1435.875 \pm 82.933 , formalin $1221.438 \pm 107.257$; Original Weber fraction, day 1 : saline $0.217 \pm 0.059$, formalin $0.222 \pm 0.054$; day 2 : saline $0.231 \pm 0.063$, formalin $0.254 \pm 0.038$; day 3 : saline $0.231 \pm 0.048$, formalin $0.222 \pm 0.032$ ). Independentsample $t$ tests showed that the formalin treatment tended to reduce the change of PSE on postinjection days $2[t(9.353)=2.143, p=0.060]$ and $3[t(8.527)=2.238, p=0.054]$, see Fig. $4 \mathrm{~A}$.

Omitted trials from the three post-days (day 1: saline $6.375 \pm 3.201$, formalin $3.625 \pm 1.992$; day 2: saline $5.000 \pm 3.082$, formalin: $2.500 \pm 0.810$; day 3: saline $5.250 \pm 3.521$, formalin 1.625 \pm 0.431 ) were also analysed. Independent-sample $t$ tests revealed no post-effect of formalin treatment in the omitted trials from any day. Two-way RM ANOVA (duration * treatment) revealed that the treatment had no significant effect on response latency on any of the 3 days [treatment effect: $F(1,14) \leqslant 1.924, p \geqslant 0.187$; interaction of duration and treatment: $F(6,84)$ $\leqslant 1.862, p \geqslant 0.097$, Supplemental Fig. 3].

In the 2-8-s range, the correct response rates to anchor-duration tones did not differ significantly on post-injection day 1 ( $2 \mathrm{~s}$ : saline $100.00 \% \pm 0.00 \%$, formalin $97.96 \% \pm 1.20 \%$; 8 s: saline $94.57 \% \pm 1.67 \%$, formalin $95.45 \% \pm 1.97 \%), 2(2 \mathrm{~s}$ : saline $99.255 \pm 0.49 \%$, formalin $100.00 \% \pm 0.00 \% ; 8 \mathrm{~s}$ : saline $97.14 \% \pm 2.33 \%$, formalin $96.73 \% \pm 2.01 \%)$, or $3(2 \mathrm{~s}$ : saline

Peer) reviewing PDF | (2020:08:52137:2:0:NEW 20 Jan 2021) 
$343100.00 \% \pm 0.00 \%$, formalin $98.78 \% \pm 0.85 \% ; 8 \mathrm{~s}$ : saline $93.77 \% \pm 2.17 \%$, formalin $95.00 \% \pm$

$3442.27 \%$ ). These results indicate that the formalin treatment did not influence the animals'

345 temporal discrimination ability for the anchor durations.

346 The results of temporal bisection tasks performed in the 2-8-s range on post-injection days

347 are shown in Fig. 3D-F. Two-way RM ANOVA revealed no significant interaction of treatment

348 and duration affecting the $P_{\mathrm{L}}$ on any day [post-injection day $1: F(6,78)=0.303, p=0.934$; post-

349 injection day 2: $F(6,78)=0.700, p=0.651$; post-injection day $3: F(6,78)=0.695, p=0.654]$.

350 The effect of treatment on $P_{\mathrm{L}}$ also was not significant [post-injection day 1: $F(1,13)=0.097, p$

$351=0.761$; post-injection day $2: F(1,13)=0.265, p=0.615$; post-injection day $3: F(1,13)=$

$3520.331, p=0.575]$. Duration had a significant effect on the $P_{\mathrm{L}}$ in all tests $(p<0.001) . R^{2}$ values

353 for curve fitting ranged from 0.83 to 1 [mean, 0.95; the PSE and Weber fraction from one rat

354 were excluded because the $R^{2}$ values were low (0.6) on post-injection day 2], with no significant

355 difference between groups on any of the 3 days.

356

357

As shown in Fig 4C, D, the treatment had no significant effect on the change of PSE or

358

359

Weber fraction from baseline on any of these post-injection day (Original PSE, day 1: saline $3.427 \pm 0.216$, formalin $3.403 \pm 0.224$; day 2 : saline $3.486 \pm 0.116$, formalin $3.784 \pm 0.23$; day 3 :

360 saline $3.500 \pm 0.180$, formalin $3.800 \pm 0.419$; Original Weber fraction , day 1: saline $0.191 \pm$

361 0.026 , formalin $0.185 \pm 0.043$; day 2 : saline $0.130 \pm 0.029$, formalin $0.207 \pm 0.042$; day 3 : saline

362 $0.186 \pm 0.038$, formalin $0.260 \pm 0.056$ )

363

Omitted trials from the three post-injection days (day 1: saline $6.750 \pm 4.259$, formalin

364

365

366

367

368

369

370

371

\section{Discussion \\ Discussion}

373

374

375

376

377

378

379

380

381

$13.143 \pm 5.501$; day 2 : saline $7.875 \pm 4.673$, formalin $9.857 \pm 4.752$; day 3 : saline $8.125 \pm 4.525$, formalin $11.000 \pm 3.9$ ) were analysed. Independent-sample $t$ tests revealed no effect of the formalin treatment on omitted trials from any day. The response latency did not differ significantly on post-injection days 1 and 2 [treatment: $F(1,13) \leqslant 2.58, p \geqslant 0.132$; interaction of duration and treatment: $F(6,78) \leqslant 1.365, p \geqslant 0.239$; Supplemental Fig. 4]. Interaction of treatment and duration affecting response latency was observed for post-injection day 3 [treatment: $F(1,14)=0.913, p=0.357$; interaction of duration and treatment: $F(6,78)=2.666$, $p=0.021$; Supplemental Fig. $4 \mathrm{C}]$. A simple main-effect analysis revealed significantly more response latency in the $4-\mathrm{s}$ trials in the formalin group $(p=0.022)$.

In this study, we investigated the effect of formalin-induced pain on time estimation at two scales in rats. Plantar formalin injection lengthened subjective time perception on the injection and post-injection days in the sub- to supra-second temporal range (0.6-2.4 s), but not in the supra-second range (2-8 s).

Human and animal studies exploring how acute pain modulates time perception within relatively short temporal ranges have yielded similar results. In humans, lengthened temporal estimation of neutral stimuli has been observed with laser-induced heat pain (0.35-1.37-s stimulus duration) (Ogden et al. 2015), in a cold pressor test (0.25-0.75-s duration) (Rey et al. 2017), with electro-cutaneous stimuli rated as inducing a high level of pain (0.24-1.30-s 
382

383

384

385

386

387

388

389

390

391

392

393

394

395

396

397

398

399

400

401

402

403

404

405

406

407

408

409

410

411

412

413

414

415

416

417

418

419

420

421

duration) (Piovesan et al. 2019), and in children with migraine (1.5-1.9-s duration) (Vicario et al. 2014). In rats, formalin-induced pain has been shown to increase temporal perception during temporal bisection task with to-be-timed tones in the 1.2-2.4-s range (Liu et al. 2019). Generally, the results of the present study are consistent with previous findings: acute pain lengthened time perception over short $(<2.4-\mathrm{s})$ durations. We found that formalin injection into the hind paw induced more nociceptive behaviours during the whole task period $(70 \mathrm{~min})$, suggesting that the animals felt pain while performing the task. This result is in agreement with previous findings that subcutaneous formalin injection induced obvious and persistent nociceptive behaviours (Jiang et al. 2014; Johansen et al. 2001). We observed a negative relationship between the duration of nociceptive behaviour and the PSE, an index of subjective duration, indicating that subjective duration lengthened with increased nociceptive behaviour. Pain is believed to influence psychological processes such as motivation (Schwartz et al. 2014) and attention (Freitas et al. 2015), which are related to temporal bisection task performance. In the sessions conducted with to-be-timed tones in the 0.6-2.4-s range, we found no difference in the number of omitted trials (a measure of motivation) or the response latency (a measure of attention). These results indicate that the rats' motivation and attention remained normal after formalin injection. Thus, we ruled out these factors as the cause of temporal overestimation in the 0.6-2.4$\mathrm{s}$ range. This perceptual effect for short-duration has been related to high arousal (self-reported and quantified by skin conductance and heart rate) in humans (Angrilli et al. 1997; Mella et al. 2011). Thus, acute pain-induced high arousal may mediate temporal overestimation in this duration range.

Few studies have examined the effect of pain on temporal perception over long durations, perhaps because the perceptual process is more complex than for short durations. Subjects must focus their attention on to-be-timed stimuli for a long time, necessitating greater involvement of cognitive abilities such as selective and sustained attention and working memory (Droit-Volet 2013; Fraisse 1984). In addition, human subjects may adopt strategies such as counting to estimate long stimulus durations (Thönes \& Hecht 2017), meaning that the process is not purely perceptual. Healthy subjects who were required to put their hands in cold $\left(7^{\circ} \mathrm{C}\right)$ water (pain condition) or warm $\left(35^{\circ} \mathrm{C}\right)$ water (control condition) for 120 or $300 \mathrm{~s}$ retrospectively underestimated these durations of pain relative to controls (Hellstrom \& Carlsson 1997; Thorn \& Hansell 1993). In contrast, Khoshnejad et al. (Khoshnejad et al. 2017a) found that healthy subjects reported longer subjective durations of a high-intensity thermal stimulus (rated as high pain, 10-11 s) than of a low-intensity thermal stimulus (rated as low pain). The 2-8-s range is used commonly in animal studies of time perception. Using the temporal bisection task in this range, Kamada and Hata (Kamada \& Hata 2018; Kamada \& Hata 2019) found that rats underestimated the duration of an auditory cue paired with an electric foot shock $(0.4 \mathrm{~mA})$, whereas Meck (Meck 1983) observed that an electric foot shock (0.2 mA) induced temporal overestimation in rats. The intensities of the electrical stimuli used in those studies may not have been sufficient to induce nociception, and the authors did not provide nociceptive data. Overall, the effect of pain on time perception in this range remains unclear. 
422

423

424

425

426

427

428

429

430

431

432

433

434

435

436

437

438

439

440

441

442

443

444

445

446

447

448

449

450

451

452

453

454

455

456

457

458

459

460

461

In the 2-8-s range, we found that $P_{\mathrm{L}} \mathrm{s}$ to intermediate-duration tones were greater in the formalin group than in the saline group, with no significant difference in the PSE. These results indicate that formalin-induced pain had a weaker effect in this range relative to the 0.6-2.4-s range. In addition, pain is known to automatically attract attention (Baliki \& Apkarian 2015). Nociceptive stimulus-induced pain has been shown to disrupt attention-based cognitive tasks in animals (Boyette-Davis et al. 2008; Freitas et al. 2015; Pais-Vieira et al. 2012), and the distraction of attention from to-be-timed stimuli has been found to shorten subjective stimulus durations in humans (Casini \& Macar 1997; Chen \& O'Neill 2001; Macar et al. 1994) and animals (Buhusi \& Meck 2006; Buhusi \& Meck 2009). In addition, we found that formalin treatment increased the response latency in intermediate-duration trials (4 and $5.04 \mathrm{~s})$ in this range, but not in the 0.6-2.4-s range. Theoretically, animals perceive long-duration tones by focusing their attention on them for longer periods. Thus, we believe that the distraction caused by pain interferes with attention during time processing, leading to the prolongation of response latency. The influence of pain may differ according to the stimulus duration; the perception of short-duration may not require the involvement of attention and could be lengthened by paininduced high arousal, whereas the perception of long-duration may be affected jointly by such arousal and attentional distraction, leading to a weaker lengthening effect in the 2-8-s range.

A post-injection effect of formalin-induced pain, namely the overestimation of tone duration, was observed only in the 0.6-2.4-s range on the second and third days after injection. We suggest that the affective component of formalin-induced pain contributed to the observed postinjection effect. Minor change was also observed after injection of normal saline. Normal saline injection only produced a transient and very mild nociceptive responses (less than $5 \mathrm{~min}$ ) and did not affect the temporal bisection task on the injection day. Therefore, these less significant changes in saline group may be due to training effects or random fluctuations. Subcutaneous formalin injection into the rat hind paw is a classic method for the creation of a model of acute inflammatory pain. Low-dose (e.g., 1\%) formalin induces phase-I and phase-II nociceptive behaviours that do not last until the following day (Fu et al. 2000; Johnston et al. 2012). However, formalin-induced pain can induce conditioned place avoidance within a few days in rats, suggesting the induction of pain-related aversion (Jiang et al. 2014; Johansen et al. 2001). Aversion and fear can induce temporal overestimation (Buetti \& Lleras 2012; Gil \& Droit-Volet 2009; Gil \& Droit-Volet 2012; Grommet et al. 2011; Watts \& Sharrock 1984), and pain-related aversion may have a similar effect. Emotional stimuli have been shown to have strong effects on perception of shorter-duration (ca. 2-s), but not longer-duration (4-6 s) (Angrilli et al. 1997; Noulhiane et al. 2007). These studies support our explanation that the overestimation of the 0.6$2.4 \mathrm{~s}$ range after formalin injection may be due to emotional dimension of pain. We did not observe the significant post-injection effect of formalin-induced pain in 2-8-s range. However, other studies have shown that conditioned fear cues induce temporal underestimation in the 2-8s range (Kamada \& Hata 2018; Kamada \& Hata 2019).

Formalin-induced acute pain did not affect temporal sensitivity in the sub-supra-second or supra-second range in this study. Previous research has revealed a negative relationship between 
462 the level of chronic stress and temporal sensitivity (measured with a temporal bisection task) in 463 healthy human subjects (Yao et al. 2015). Acute psychosocial stress may induce a stronger 464 cortisol response, but may not significantly affect temporal sensitivity (Yao et al. 2016).

465 Decreased temporal sensitivity has been observed in patients with psychiatric disorders, such as 466 Parkinson's disease and schizophrenia (Alústiza et al. 2016; Harrington et al. 2011; Jahanshahi et 467 al. 2010), suggesting an association with pathological changes in the brain. Our model of 468 formalin-induced acute pain may not have been sufficient to induce pathological changes in 469 brain function.

470

471

472

473

474

475

476

477

478

479

480

481

482

483

484

485

486

487

488

489

490

491

492

\section{Conclusions}

494

495

496

497

498

499

500

Our results show that formalin-induced pain affected time perception in the sub- to suprasecond range on days after formalin injection, but we conducted observations on only three such days. Thus, the duration of effect persistence remains unclear. Previous studies have shown that animals can learn to be unresponsive to intermediate-duration after repeated performance of the temporal bisection task (Brown et al. 2011). To avoid the omission of an excessive number of trials, we measured time perception in only five consecutive test sessions. Despite this limitation, the present study can provide guidance for further research. For example, combining formalin pain induced conditioned placed aversion paradigm with time perception task can obtain pain emotion related behavioral results and time perception data at the same time, which will clarify the impact of pain emotion on time perception.

This study explored the optimal time window for pain to affect time perception, and partially confirmed the possible role of pain emotion in it. These results can provide evidence for some theoretical models of time perception. Striatal beat frequency (SBF) model emphasizes the critical role of cortical activities in temporal information processing (van Rijn et al. 2014). As a multidimensional and complex experience, pain can activate many brain regions, including emotion related brain areas, such as anterior cingulate cortex and anterior insular; sensory related brain areas, such as primary somatosensory cortex and lateral thalamus; cognitive related brain areas, such as medial prefrontal cortex (Wiech 2016). The medial prefrontal cortex has been shown to play a key role in the encoding of temporal information (Emmons et al. 2017; Xu et al. 2014). Although the influence of other pain related cortical regions on time perception coding remains to be explored, we can still believe that exploring the impact of pain on time perception and its mechanism will help support the SBF model and even help to establish a more optimized physiological model of time perception.

This study showed that the effect of acute pain on time perception depends on the duration of the tone to be estimated. Acute pain leads to more significant overestimation of shorterduration than of longer-duration. Pain-induced high arousal and distraction may jointly affect time perception, and this effect may differ between the short-term and long-term ranges. In addition, the emotional component of pain may play a role independent of the sensory component. In future research, the effects of the emotional processing of pain on temporal perception should be explored further.

Peer) reviewing PDF | (2020:08:52137:2:0:NEW 20 Jan 2021) 


\section{References}

502 Alústiza I, Radua J, Albajes-Eizagirre A, Domínguez M, Aubá E, and Ortuño F. 2016. Meta-Analysis of Functional

503

504

505

506

507

508

509

510

511

512

513

514

515

516

517

518

519

520

521

522

523

524

525

526

527

528

529

530

531

532

533

534

535

536

537

538

539

540

541

542

543

544

545

546

547

548

549

550 Neuroimaging and Cognitive Control Studies in Schizophrenia: Preliminary Elucidation of a Core Dysfunctional Timing Network. Front Psychol 7:192. 10.3389/fpsyg.2016.00192

Angrilli A, Cherubini P, Pavese A, and Manfredini S. 1997. The influence of affective factors on time perception. Perception and Psychophysics 59:972-982. 10.3758/BF03205512

Baliki MN, and Apkarian AV. 2015. Nociception, Pain, Negative Moods, and Behavior Selection. Neuron 87:474-491. 10.1016/j.neuron.2015.06.005

Barr GA. 1998. Maturation of the biphasic behavioral and heart rate response in the formalin test. Pharmacol Biochem Behav 60:329-335.

Boyette-Davis JA, Thompson CD, and Fuchs PN. 2008. Alterations in attentional mechanisms in response to acute inflammatory pain and morphine administration. Neuroscience 151:558-563. 10.1016/j.neuroscience.2007.10.032

Brasted PJ, Humby T, Dunnett SB, and Robbins TW. 1997. Unilateral Lesions of the Dorsal Striatum in Rats Disrupt Responding in Egocentric Space. Journal of Neuroscience 17:8919-8926. 10.1523/JNEUROSCI.17-2208919.1997

Brown BL, Höhn S, Faure A, von Hörsten S, Le Blanc P, Desvignes N, El Massioui N, and Doyère V. 2011. Temporal sensitivity changes with extended training in a bisection task in a transgenic rat model. Front Integr Neurosci 5:44. 10.3389/fnint.2011.00044

Buetti S, and Lleras A. 2012. Perceiving control over aversive and fearful events can alter how we experience those events: an investigation of time perception in spider-fearful individuals. Front Psychol 3:337. 10.3389/fpsyg.2012.00337

Buhusi CV, and Meck WH. 2006. Time sharing in rats: A peak-interval procedure with gaps and distracters. Behav Processes 71:107-115. 10.1016/j.beproc.2005.11.017

Buhusi CV, and Meck WH. 2009. Relative time sharing: new findings and an extension of the resource allocation model of temporal processing. Philosophical Transactions of the Royal Society of London Series B: Biological Sciences 364:1875-1885. 10.1098/rstb.2009.0022

Callu D, El Massioui N, Dutrieux G, Brown BL, and Doyere V. 2009. Cognitive processing impairments in a suprasecond temporal discrimination task in rats with cerebellar lesion. Neurobiol Learn Mem 91:250-259. 10.1016/j.nlm.2008.12.002

Casini L, and Macar F. 1997. Effects of attention manipulation on judgments of duration and of intensity in the visual modality. Mem Cognit 25:812-818.

Chen Z, and O'Neill P. 2001. Processing demand modulates the effects of spatial attention on the judged duration of a brief stimulus. Percept Psychophys 63:1229-1238.

Church RM, and Deluty MZ. 1977. Bisection of temporal intervals. J Exp Psychol Anim Behav Process 3:216-228. 10.1037//0097-7403.3.3.216

Crystal JD, Maxwell KW, and Hohmann AG. 2003. Cannabinoid modulation of sensitivity to time. Behavioural Brain Research 144:57-66. 10.1016/S0166-4328(03)00062-7

Deane AR, Millar J, Bilkey DK, and Ward RD. 2017. Maternal immune activation in rats produces temporal perception impairments in adult offspring analogous to those observed in schizophrenia. PLoS One 12:e0187719. 10.1371/journal.pone.0187719

Droit-Volet S. 2013. Time perception, emotions and mood disorders. J Physiol Paris 107:255-264. 10.1016/j.jphysparis.2013.03.005

Emmons EB, De Corte BJ, Kim Y, Parker KL, Matell MS, and Narayanan NS. 2017. Rodent Medial Frontal Control of Temporal Processing in the Dorsomedial Striatum. The Journal of Neuroscience 37:8718-8733. 10.1523/jneurosci.1376-17.2017

Fayolle S, Gil S, and Droit-Volet S. 2015. Fear and time: Fear speeds up the internal clock. Behav Processes 120:135140. 10.1016/j.beproc.2015.09.014

Fraisse P. 1984. Perception and estimation of time. Annu Rev Psychol 35:1-36. 10.1146/annurev.ps.35.020184.000245 
551

552

553

554

555

556

557

558

559

560

561

562

563

564

565

566

567

568

569

570

571

572

573

574

575

576

577

578

579

580

581

582

583

584

585

586

587

588

589

590

591

592

593

594

595

596

597

598

599

600

601

602
Freitas KC, Hillhouse TM, Leitl MD, and Negus SS. 2015. Effects of acute and sustained pain manipulations on performance in a visual-signal detection task of attention in rats. Drug Dev Res 76:194-203. 10.1002/ddr.21255

Fu KY, Light AR, and Maixner W. 2000. Relationship between nociceptor activity, peripheral edema, spinal microglial activation and long-term hyperalgesia induced by formalin. Neuroscience 101:1127-1135. 10.1016/S0306-4522(00)00376-6

Gibbon J, Church RM, and Meck WH. 1984. Scalar timing in memory. Annals of the New York Academy of Sciences 423:52.

Gil S, and Droit-Volet S. 2009. Time perception, depression and sadness. Behav Processes 80:169-176. 10.1016/j.beproc.2008.11.012

Gil S, and Droit-Volet S. 2012. Emotional time distortions: the fundamental role of arousal. Cogn Emot 26:847-862. 10.1080/02699931.2011.625401

Grommet EK, Droit-Volet S, Gil S, Hemmes NS, Baker AH, and Brown BL. 2011. Time estimation of fear cues in human observers. Behav Processes 86:88-93. 10.1016/j.beproc.2010.10.003

Hare RD. 1963. The estimation of short temporal intervals terminated by shock. J Clin Psychol 19:378-380.

Harrington DL, Castillo GN, Greenberg PA, Song DD, Lessig S, Lee RR, and Rao SM. 2011. Neurobehavioral mechanisms of temporal processing deficits in Parkinson's disease. PLoS One 6:e17461. 10.1371/journal.pone.0017461

Hellstrom C, and Carlsson SG. 1997. Busy with pain: disorganization in subjective time in experimental pain. Eur J Pain 1:133-139.

Huang S, Qiu J, Liu P, Li Q, and Huang X. 2018. The Effects of Same- and Other-Race Facial Expressions of Pain on Temporal Perception. Front Psychol 9:2366. 10.3389/fpsyg.2018.02366

Jahanshahi M, Jones CR, Zijlmans J, Katzenschlager R, Lee L, Quinn N, Frith CD, and Lees AJ. 2010. Dopaminergic modulation of striato-frontal connectivity during motor timing in Parkinson's disease. Brain 133:727-745. 10.1093/brain/awq012

Jiang Z-C, Pan Q, Zheng C, Deng X-F, Wang J-Y, and Luo F. 2014. Inactivation of the prelimbic rather than infralimbic cortex impairs acquisition and expression of formalin-induced conditioned place avoidance. Neuroscience Letters 569:89-93. 10.1016/j.neulet.2014.03.074

Johansen JP, Fields HL, and Manning BH. 2001. The Affective Component of Pain in Rodents: Direct Evidence for a Contribution of the Anterior Cingulate Cortex. Proceedings of the National Academy of Sciences of the United States of America 98:8077-8082. 10.1073/pnas.141218998

Johnston IN, Maier SF, Rudy JW, and Watkins LR. 2012. Post-conditioning experience with acute or chronic inflammatory pain reduces contextual fear conditioning in the rat. Behavioural Brain Research 226:361368. 10.1016/j.bbr.2011.08.048

Kamada T, and Hata T. 2018. Insular cortex inactivation generalizes fear-induced underestimation of interval timing in a temporal bisection task. Behav Brain Res 347:219-226. 10.1016/j.bbr.2018.03.016

Kamada T, and Hata T. 2019. Basolateral amygdala inactivation eliminates fear-induced underestimation of time in a temporal bisection task. Behav Brain Res 356:227-235. 10.1016/j.bbr.2018.07.027

Khoshnejad M, Khoshnejad M, Roy M, Roy M, Martinu K, Martinu K, Chen J-I, Chen J-I, Cohen-Adad J, Cohen-Adad J, Grondin S, Grondin S, Rainville P, and Rainville P. 2017a. Brain processing of the temporal dimension of acute pain in short-term memory. Pain 158:2001-2011. 10.1097/j.pain.0000000000001003

Khoshnejad M, Roy M, Martinu K, Chen JI, Cohen-Adad J, Grondin S, and Rainville P. 2017b. Brain processing of the temporal dimension of acute pain in short-term memory. Pain 158:2001-2011. 10.1097/j.pain.0000000000001003

Liu X, Wang N, Wang J, and Luo F. 2019. Formalin-induced and neuropathic pain altered time estimation in a temporal bisection task in rats. Scientific Reports 9:1-11. 10.1038/s41598-019-55168-w

Macar F, Grondin S, and Casini L. 1994. Controlled attention sharing influences time estimation. Mem Cognit 22:673-686.

McClure EA, Saulsgiver KA, and Wynne CD. 2005. Effects of D-amphetamine on temporal discrimination in pigeons. Behav Pharmacol 16:193-208.

Meck WH. 1983. Selective adjustment of the speed of internal clock and memory processes. J Exp Psychol Anim Behav Process 9:171-201.

PeerJ reviewing PDF | (2020:08:52137:2:0:NEW 20 Jan 2021) 
603

604

605

606

607

608

609

610

611

612

613

614

615

616

617

618

619

620

621

622

623

624

625

626

627

628

629

630

631

632

633

634

635

636

637

638

639

640

641

642

643

644

645

646

647

648

649

650

651

652

653

Mella N, Conty L, and Pouthas V. 2011. The role of physiological arousal in time perception: psychophysiological evidence from an emotion regulation paradigm. Brain Cogn 75:182-187. 10.1016/j.bandc.2010.11.012

Melzack R, and Wall PD. 1965. Pain mechanisms: a new theory. Science 150:971-979.

Noulhiane M, Mella N, Samson S, Ragot R, and Pouthas V. 2007. How Emotional Auditory Stimuli Modulate Time Perception. Emotion 7:697-704. 10.1037/1528-3542.7.4.697

Ogden RS, Moore D, Redfern L, and McGlone F. 2015. The effect of pain and the anticipation of pain on temporal perception: A role for attention and arousal. Cogn Emot 29:910-922. 10.1080/02699931.2014.954529

Orduña V, Hong E, and Bouzas A. 2011. Timing behavior in streptozotocin-induced diabetic rats. Behav Brain Res 224:189-194. 10.1016/j.bbr.2011.06.005

Pais-Vieira M, Aguiar P, Lima D, and Galhardo V. 2012. Inflammatory pain disrupts the orbitofrontal neuronal activity and risk-assessment performance in a rodent decision-making task. Pain 153:1625-1635. 10.1016/j.pain.2012.04.011

Piovesan A, Mirams L, Poole H, Moore D, and Ogden R. 2019. The relationship between pain-induced autonomic arousal and perceived duration. Emotion 19:1148-1161. 10.1037/emo0000512

Rey AE, Michael GA, Dondas C, Thar M, Garcia-Larrea L, and Mazza S. 2017. Pain dilates time perception. Sci Rep 7:15682. 10.1038/s41598-017-15982-6

Santuzzi CH, Neto Hde A, Pires JG, Goncalves WL, Gouvea SA, and Abreu GR. 2013. High-frequency transcutaneous electrical nerve stimulation reduces pain and cardio-respiratory parameters in an animal model of acute pain: participation of peripheral serotonin. Physiother Theory Pract 29:630-638. 10.3109/09593985.2013.774451

Schwartz N, Temkin P, Jurado S, Lim BK, Heifets BD, Polepalli JS, and Malenka RC. 2014. Chronic pain. Decreased motivation during chronic pain requires long-term depression in the nucleus accumbens. Science 345:535542. 10.1126/science.1253994

Soares S, Atallah BV, and Paton JJ. 2016. Midbrain dopamine neurons control judgment of time. Science 354:12731277. 10.1126/science.aah5234

Thönes S, and Hecht H. 2017. Counting does not improve the accuracy of long time productions. Atten Percept Psychophys 79:2576-2589. 10.3758/s13414-017-1407-5

Thorn BE, and Hansell PL. 1993. Goals for coping with pain mitigate time distortion. Am J Psychol 106:211-225.

Tipples J. 2011. When time stands still: fear-specific modulation of temporal bias due to threat. Emotion 11:74-80. $10.1037 / \mathrm{a} 0022015$

Treisman M. 1963. Temporal discrimination and the indifference interval. Implications for a model of the "internal clock". Psychol Monogr 77:1-31. 10.1037/h0093864

van Rijn H, Gu BM, and Meck WH. 2014. Dedicated clock/timing-circuit theories of time perception and timed performance. Adv Exp Med Biol 829:75-99. 10.1007/978-1-4939-1782-2_5

Vicario CM, Gulisano M, Martino D, and Rizzo R. 2014. The perception of time in childhood migraine. Cephalalgia 34:548-553. 10.1177/0333102413517774

Ward RD, and Odum AL. 2007. Disruption of temporal discrimination and the choose-short effect. Learn Behav 35:60-70.

Watts FN, and Sharrock R. 1984. Fear and time estimation. Percept Mot Skills 59:597-598. 10.2466/pms.1984.59.2.597

Wiech K. 2016. Deconstructing the sensation of pain: The influence of cognitive processes on pain perception. Science 354:584-587. 10.1126/science.aaf8934

Wiech K, and Tracey I. 2013. Pain, decisions, and actions: a motivational perspective. Front Neurosci 7:46. 10.3389/fnins.2013.00046

Xu M, Zhang SY, Dan Y, and Poo MM. 2014. Representation of interval timing by temporally scalable firing patterns in rat prefrontal cortex. Proc Natl Acad Sci U S A 111:480-485. 10.1073/pnas.1321314111

Yao Z, Wu J, Zhou B, Zhang K, and Zhang L. 2015. Higher chronic stress is associated with a decrease in temporal sensitivity but not in subjective duration in healthy young men. Front Psychol 6:1010. 10.3389/fpsyg.2015.01010

Yao Z, Zhang L, Jiang C, Zhang K, and Wu J. 2016. Stronger cortisol response to acute psychosocial stress is correlated with larger decrease in temporal sensitivity. PeerJ 4:e2061. 10.7717/peerj.2061

654

Peer) reviewing PDF | (2020:08:52137:2:0:NEW 20 Jan 2021) 
Figure 1

Effect of formalin injection on temporal bisection behaviours in the 0.6-2.4-s range.

(A-C) Nociceptive behaviours observed during the temporal bisection task (70 $\mathrm{min}$ ) and in phases I and II after treatment. (D, E) Best-fitting curves for baseline and the injection day, respectively. ( $F, G)$ Average PSEs and Weber fractions for the two groups at baseline and on the injection day, respectively. $(\mathrm{H})$ Correlation between PSEs and total durations of nociceptive behaviours on the injection day. ${ }^{*} p<0.05,{ }^{* *} p<0.01$, ${ }^{* *} p<0.001$ vs. saline group; $\# p<0.05$ vs. baseline.
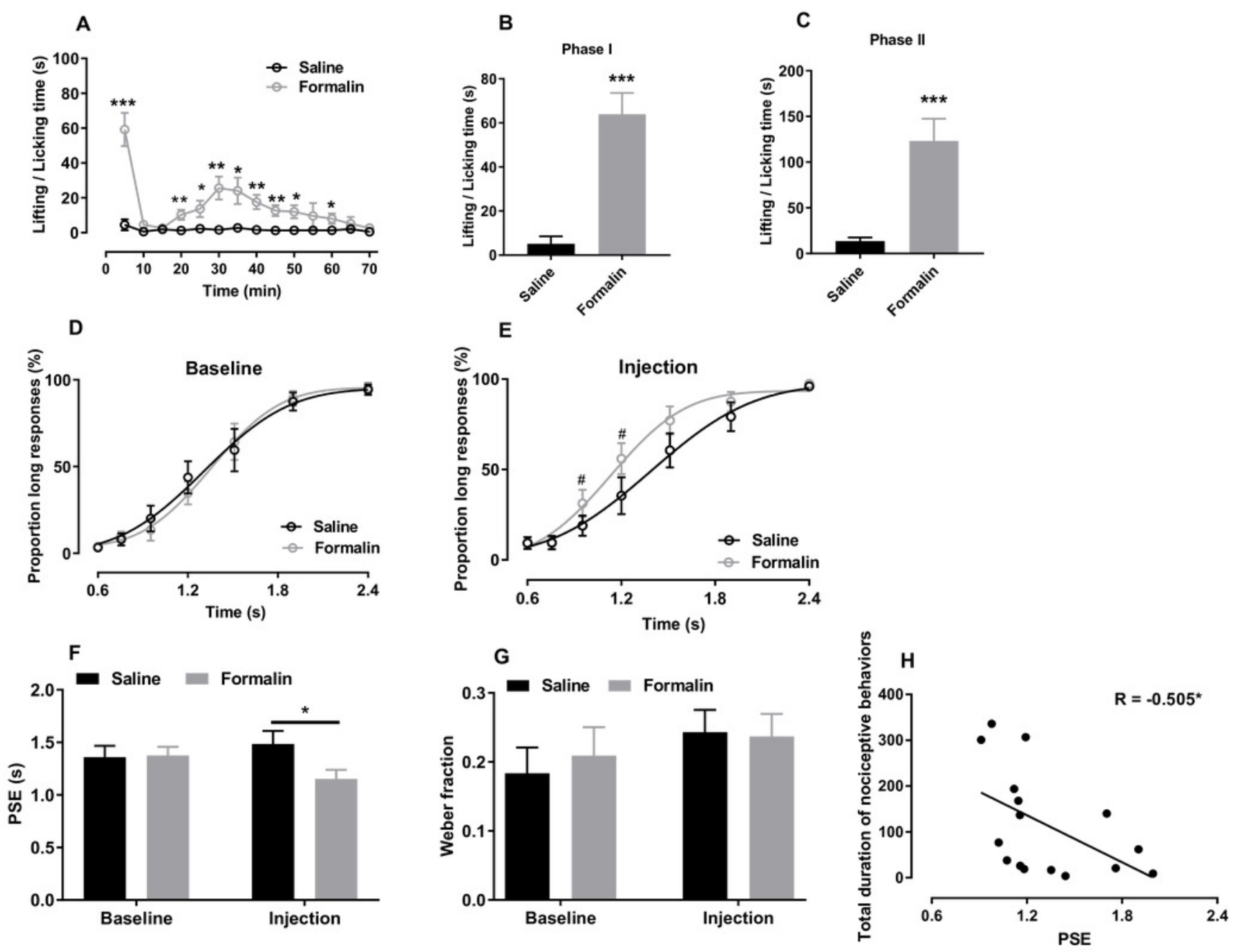


\section{Figure 2}

Effect of formalin injection on temporal bisection task performance in the 2-8-s range.

(A-C) Nociceptive behaviours observed during the temporal bisection task (70 min)and in phases I and II after treatment(D, E) Best-fitting curves for baseline and the injection day, respectively. $(F, G)$ Average PSEs and Weber fractions for the two groups at baseline and on the injection day, respectively. $(\mathrm{H})$ Correlation between PSEs and total durations of nociceptive behaviours on the injection day. ${ }^{*} p<0.05,{ }^{* *} p<0.01,{ }^{* * *} p<0.001$ vs. saline group. 
A
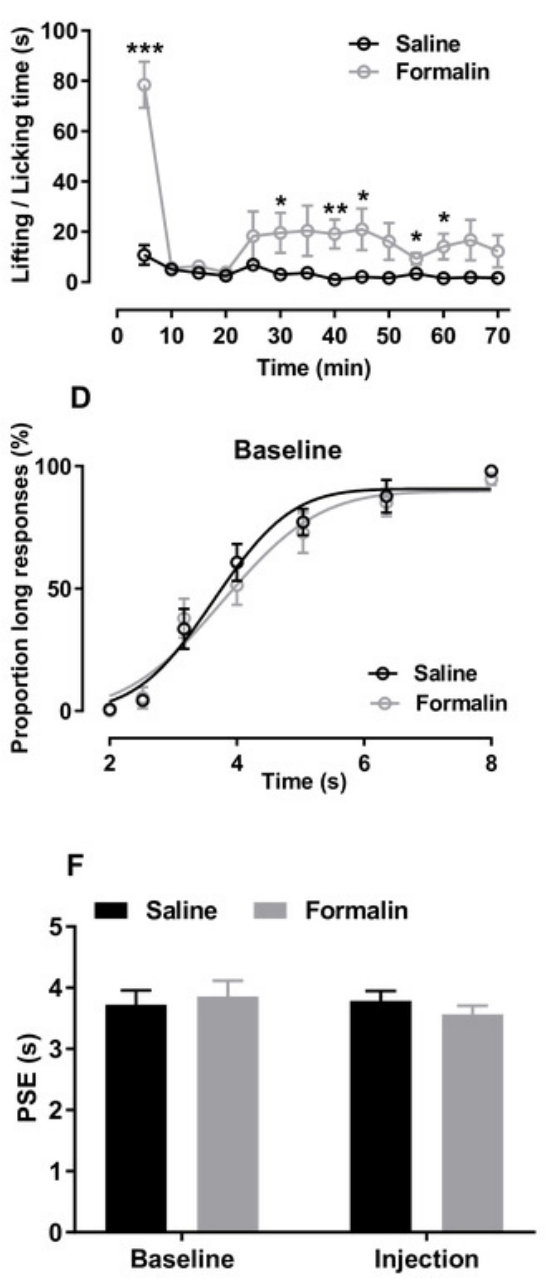

B
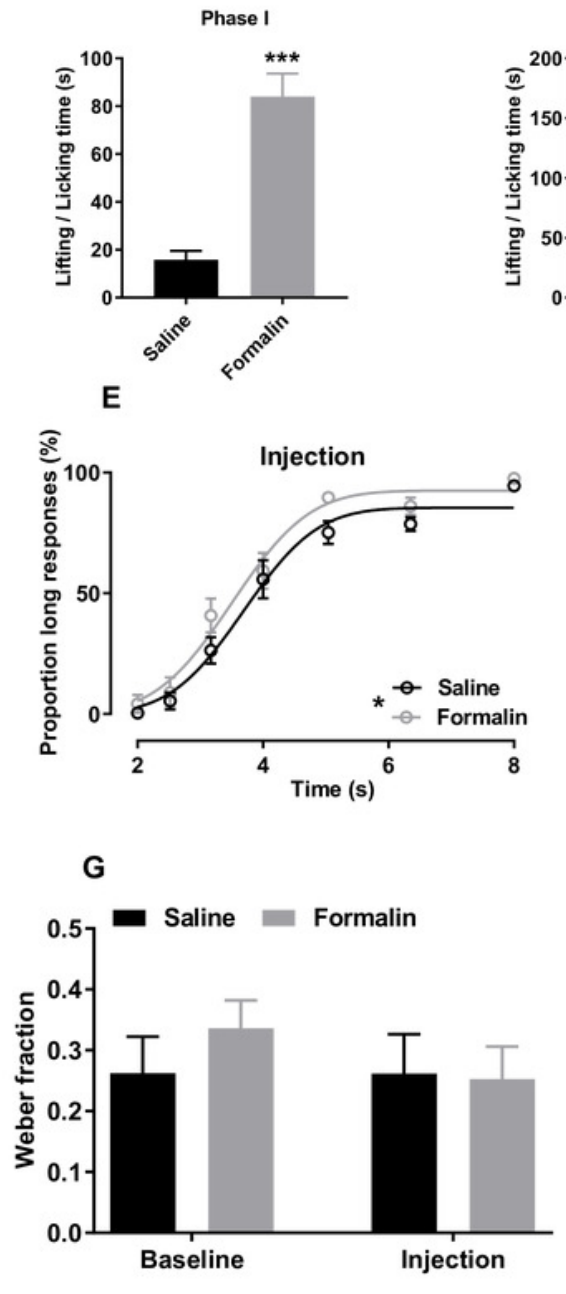

C

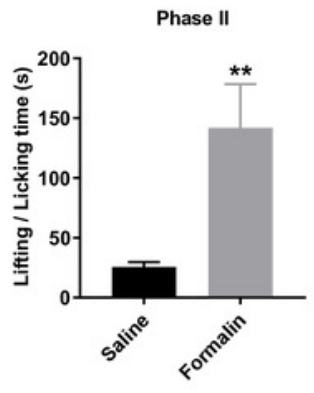

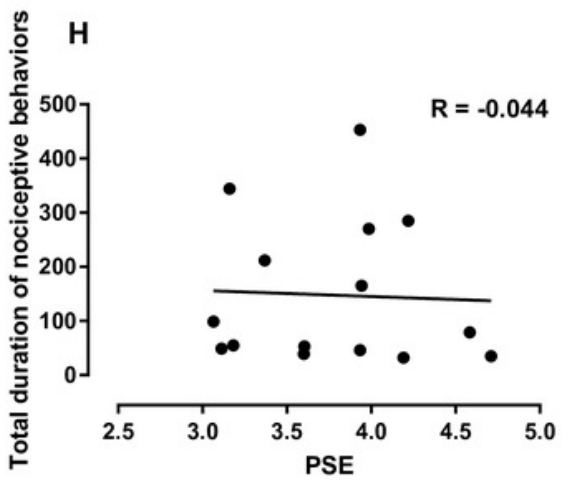


Figure 3

Post-injection effect of formalin treatment on temporal bisection task performance in the $0.6-2.4-5$ and $2-8-\mathrm{s}$ ranges.

(A-C) Average PLs for to-be-timed tone durations in the 0.6-2.4-s range and best-fitting curves for post-injection days 1-3, respectively. (D-F) Results of temporal bisection tasks in the 2-8-s range on post-injection days 1-3, respectively.
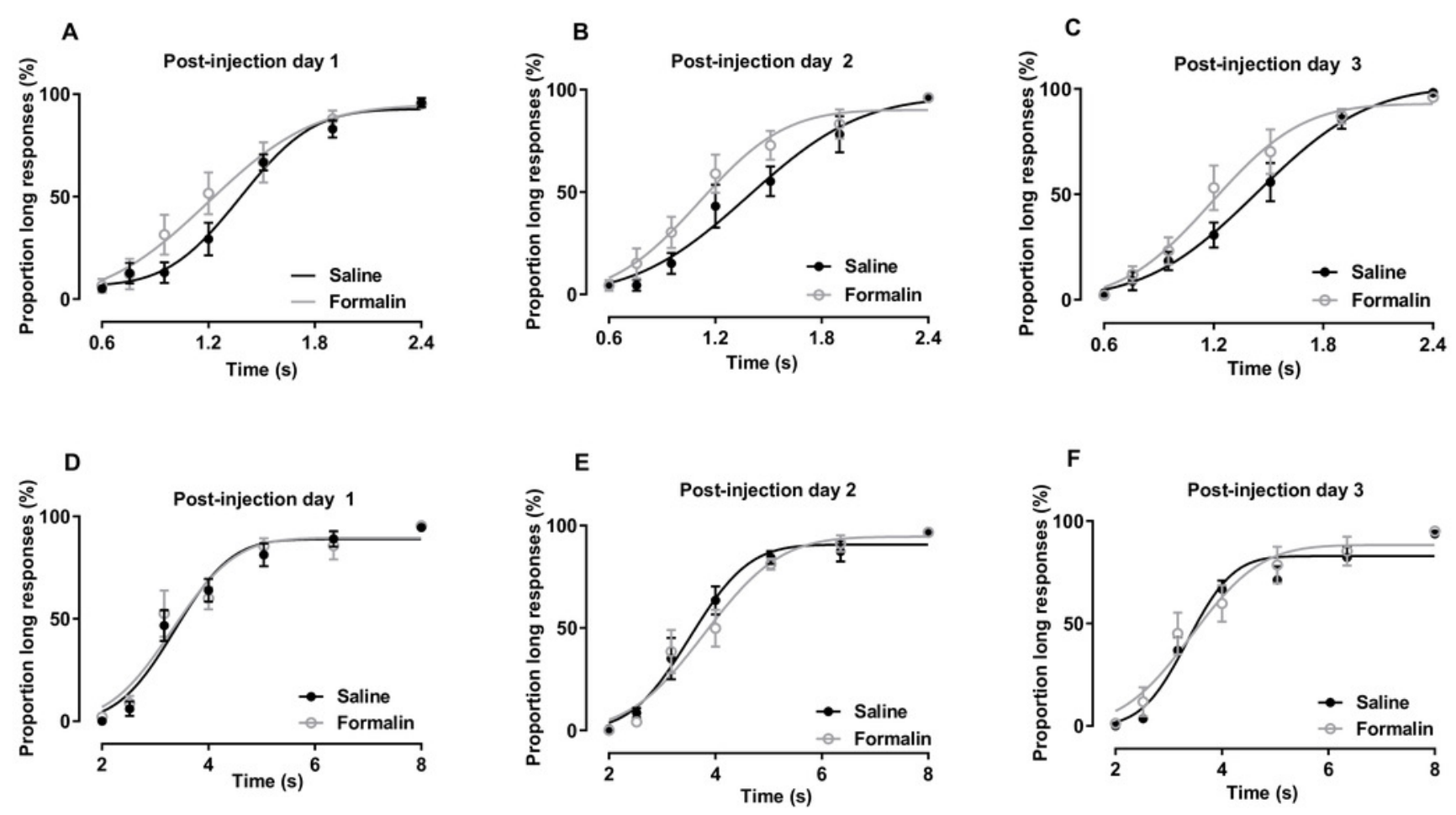
Figure 4

Changes in PSE and the Weber fraction from baseline on post-injection days 1-3.

Average changes in PSE and the Weber fraction for temporal tasks in the 0.6-2.4-s (A, B) and 2-8-s (C, D) ranges.
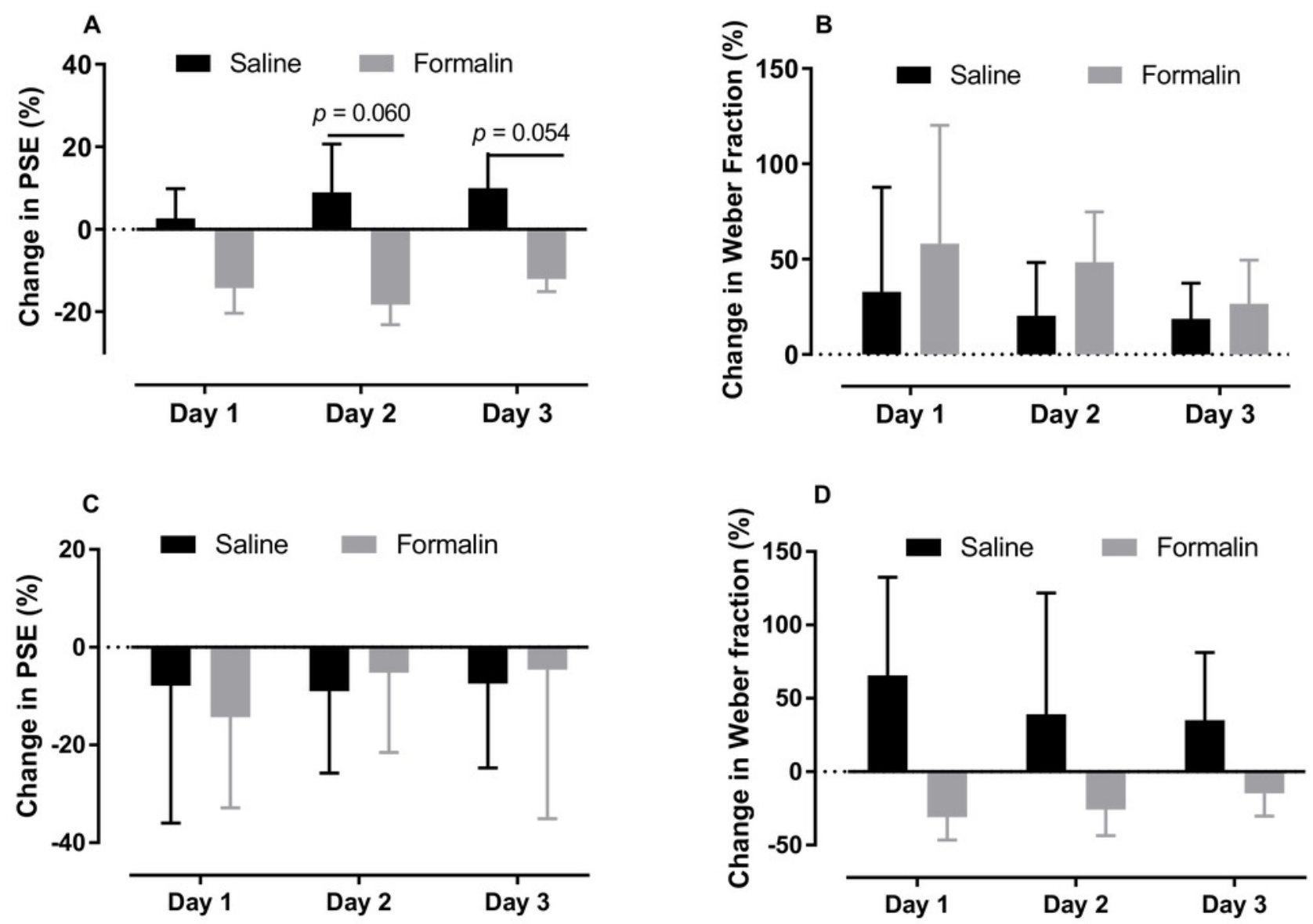